\title{
Successful management of pregnancy and delivery of a patient with chronic myeloid leukaemia (CML) without active intervention
}

\author{
R K L S Rajapaksha ${ }^{1}$, W M Y S Bandara ${ }^{2}$, E A J Manori ${ }^{2}$ \\ Sri Lanka Journal of Obstetrics and Gynaecology 2012; 34: 21-22
}

\begin{abstract}
Even though uncommon the management of a malignant disease during pregnancy is a clinical challenge to the obstetrician as a favourable outcome is expected for both the mother and baby. We report a successful management of a 21-year old mother, who was in her first pregnancy and found to be in the chronic phase of chronic myeloid leukaemia (CML) in the second trimester of her pregnancy, at DGH, Nuwaraeliya.
\end{abstract}

Key words: CML, chronic phase, hydroxyurea.

\section{Introduction}

Chronic myeloid leukaemia is an uncontrolled proliferation of myeloid cells. Its diagnosis is established by identifying clonal expansion of haematopoietic stem cells possessing a reciprocal translocation between chromosome 9 and 22 or Philadelphia translocation which is found in $90 \%$ 95\% patients with CML. If untreated CML follows the natural course from chronic phase to accelerated phase and ultimately ends up with blast crisis with mean life expectancy of 4 years ${ }^{1}$.

In the rare event in which a woman is newly diagnosed with CML during pregnancy, with an incidence of $1: 75,000^{2}$, observation and close monitoring are appropriate initial strategies as the management of these patients. For most women with chronic phase disease, initiation of treatment can be delayed until after delivery and cessation of breast feeding if appropriate. Several strategies, including interferon alpha, hydroxyurea and aphaeresis have been suggested for the management of leukocytosis (WBC count $\left.>100 \times 10^{9} / \mathrm{L}\right)$ and/or thrombocytosis (platelets $>500 \times 10^{9} / \mathrm{L}$ ) in these patients ${ }^{3}$. Imatinib is now the standard therapy for patients with CML, and

\footnotetext{
${ }^{1}$ Consultant Obstetrician and Gynaecologist,

${ }^{2}$ House Officer in Obstetrics and Gynaecology, DGH Nuwaraeliya, Sri Lanka.

Correspondence: Lasantha Sanjeewa Rajapaksha

E-mail: lasanthajani@yahoo.com
}

there are multiple case reports in the literature of women being treated for CML with imatinib who then discovered pregnancy after some time of exposure to the drug4.

\section{Case history}

A 28-year old woman was referred at 28 weeks from antenatal clinic because of high white blood cell (WBC) count while being investigated for urinary tract infection. Other than mild epigastric pain she was asymptomatic on initial presentation. She had an anomaly scan by 20 weeks which revealed no gross foeto/placental abnormalities. On initial examination she had no hepatomegally. Initial WBC count was $65 \times 10^{9} / \mathrm{L}$ with neutrophil $-53 \%$, blasts $1 \%$, megacaryocytes - $14 \%$, myelocytes $-15 \%$, lymphocytes $-7 \%$, eosinophils $-4 \%$, monocytes $-2 \%$, basophils $-2 \%, \mathrm{Hb}-12.2 \mathrm{~g} / \mathrm{dl}$ and platelet count was $365 \times 10^{9} / \mathrm{L}$. Blood picture showed features suggestive of myeloproliferative disorder most likely CML. Bone marrow aspiration biopsy revealed markedly hyperplastic with increased whole spectrum of granulocytic cell series with two peaks at mature neutrophils and myelocyte stages, blasts were being $5 \%$ out of total nucleated cells. Detection of BCRABL transcript using bone marrow sample failed. Peripheral blood sample was negative for Philadelphia chromosome.

The management of the patient was multi disciplinary with the involvement of clinical oncologist. The pregnancy was allowed to continue with close serial monitoring of WBC count, the maternal and the foetal wellbeing. On oncological advice the patient was not started on treatment as the serial WBC counts were below $100 \times 10^{9}$. The delivery was planned at 34 weeks with steroid cover for foetal lung maturity. Serial full blood counts were done and they fluctuated between 60.8 to $92.6 \times 10^{9} / \mathrm{L}$. At the time of delivery total white cell count was 76.6 $\times 10^{9} / \mathrm{L}$.

A caesarean section was performed at 34 weeks and a healthy baby girl, weighing 1900g, was delivered. Baby's neonatal examination was normal with an uneventful postnatal period but she was on formula feeds. 
Two days after delivery, hydroxyurea was started as the treatment of CML. Post partum period was uneventful other than a mild caesarean section wound infection which well responded to oral cefuroxime.

On day 5 of hydroxyurea, patient was clinically well and total WBC count was $32.3 \times 10^{9} / \mathrm{L}$ and platelet count was $410 \times 10^{9} / \mathrm{L}$. Patient is being followed up by clinical oncology department.

\section{Discussion}

The ongoing pregnancy of a patient with a neoplastic disease requiring cytotoxic treatment is a therapeutic dilemma. The effects of cytotoxic drugs on foetus may be immediate such as abortive and teratogenic or late. Late effects, which are less well known, are gonadal and other endocrinal disorders, growth and developmental problems which involve central nervous system, immunosuppression and genetic and teratogenic disorders that may affect future generations. These risks are concentrated in the first trimester and depend on chemotherapeutic agents or the combinations of the agents ${ }^{5}$.

Other conventional therapeutic options of chronic phase CML include hydroxyurea, busalfan, interferon based regimens and stem cell transplantation. Through rational drug development, imatinib, gleevac (formally STI571), a bcr-abl tyrosine kinase inhibitor, offers a new hope for expanded options for patients with $\mathrm{CML}^{5}$.

Leukapharesis has been successfully used in both acute and chronic leukaemia for the rapid reduction of WBC counts in patients with impending vascular occlusion. Since chronic mechanical cytoreduction does not prolong survival in these patients, and because it is inconvenient, costly and time consuming, leukaphresis is not currently recommended as maintenance therapy for these diseases ${ }^{5}$.

The patient reported in our case illustrates another approach which may be applicable in certain patients in pregnancy. This patient was able to continue the pregnancy up to 34 weeks, without symptoms related to disease, with close monitoring of maternal and foetal wellbeing. Similar approach has been described in an earlier case.

\section{References}

1. Wetzler m, Byrd JC, Bloomfield CD. Harrison's Principles of Internal Medicine, Oncology. 17th Edition. United States: McGraw-Hills Company: 2008.

2. Fadilah SAW, Ahmad-Zailani H, Soon-Keng C, Norlaila M. Successful treatment of chronic myeloid leukaemia during pregnancy with hydroxyurea. Leukaemia 2002; 16(6): 1202-03.

3. Leusley DM, Baker PN, Editors. Obstetrics and Gynaecology: An Evidence-based Text for MRCOG. 2nd Edition. London: Hodder Arnold Publisher (Ltd): 2010.

4. Susan C, Hagop K, Patricia A, Jorge E, Cortes. Successful Completion of pregnancy in a patient with chronic myeloid leukaemia without active intervention. Clinical Lymphoma and Myeloma 2009; 324-7.

5. Ridvan A, Fahir O, Vildan O, Tulay O, Ulku O, Yalcin K, et al. Successful pregnancy and delivery in a patient with CML and management of CML with leukapharesis in pregnancy. Jpn J Clin Oncol 2004; 34(4): 215-17. 